\title{
Neurofeedback as a Treatment Intervention in ADHD: Current Evidence and Practice
}

\author{
Stefanie Enriquez-Geppert ${ }^{1,2} \cdot$ Diede Smit $^{1} \cdot$ Miguel Garcia Pimenta ${ }^{1} \cdot$ Martijn Arns ${ }^{3,4,5}$
}

Published online: 28 May 2019

(C) The Author(s) 2019

\begin{abstract}
Purpose of Review Current traditional treatments for ADHD present serious limitations in terms of long-term maintenance of symptom remission and side effects. Here, we provide an overview of the rationale and scientific evidence of the efficacy of neurofeedback in regulating the brain functions in ADHD. We also review the institutional and professional regulation of clinical neurofeedback implementations.

Recent Findings Based on meta-analyses and (large multicenter) randomized controlled trials, three standard neurofeedback training protocols, namely theta/beta (TBR), sensori-motor rhythm (SMR), and slow cortical potential (SCP), turn out to be efficacious and specific. However, the practical implementation of neurofeedback as a clinical treatment is currently not regulated.

Summary We conclude that neurofeedback based on standard protocols in ADHD should be considered as a viable treatment alternative and suggest that further research is needed to understand how specific neurofeedback protocols work. Eventually, we emphasize the need for standard neurofeedback training for practitioners and binding standards for use in clinical practice.
\end{abstract}

Keywords Neurofeedback $\cdot$ ADHD $\cdot$ Current status $\cdot$ Brain computer interface $\cdot$ Clinical practice

\section{Introduction}

Similar to many of his 9-year-old school peers, Brian was put on psychostimulants after complaints of poor concentration and impulsivity that met ADHD diagnostic criteria. Despite a remarkable improvement in his academic performance, parent and teachers noticed a reduction in appetite and weight

This article is part of the Topical Collection on Attention-Deficit Disorder

Stefanie Enriquez-Geppert

S.Enriquez.Geppert@rug.nl

1 Department of Clinical and Developmental Neuropsychology, Faculty of Behavioural and Social Sciences, University of

Groningen, Grote Kruisstraat 2/1, 9712

TS Groningen, The Netherlands

2 Department of Biomedical Sciences of Cells \& Systems, Section of Cognitive Neuropsychiatry, University of Groningen, Groningen, The Netherlands

3 Department of Experimental Psychology, Utrecht University, Utrecht, The Netherlands

4 neuroCare Group, Munich, Germany

5 Research Institute Brainclinics, Nijmegen, The Netherlands loss after the onset of the medication. Moreover, when not under the effects of medication, inattention and impulsivity rebounded creating innumerous embarrassments to him and his family. His parents are now considering neurofeedback-a non-pharmacological and non-invasive intervention that has shown promising results in managing the ADHD symptoms in the long run and without side effects [1].

Despite being the most often applied and accepted treatments for ADHD, recent large-scale studies and meta-analyses have demonstrated limitations of psychostimulants and behavioral therapy. Thus, research and the development of nonpharmacological treatments such as neurofeedback have been recommended. To date, however, the clinical value of neurofeedback is still debated, with evaluations ranging from "efficacious and specific" $[2,3]$ to "fails to support neurofeedback as an effective treatment for ADHD". [4•] In this contribution, we will introduce neurofeedback and review the application of neurofeedback to ADHD as well as its past and current evidence in the treatment of ADHD. We will also attempt to reconcile these seemingly discrepant research findings.

Current Treatment Approaches in ADHD Several guidelines exist for the diagnosis and treatment of children who have or are suspected of having ADHD. Among these are international, 
national, and various regional guidelines for general practitioners. Additionally, there are guidelines for youth aid and youth protective services.

Traditionally, the treatment of ADHD consists of pharmacotherapy, often complemented by behavioral therapy based on parent management training and mediation training for parents and teachers [5]. Additionally, classroom interventions, academic interventions, and peer-related interventions are being used as psychosocial therapeutic approaches [6]. Regarding pharmacotherapy, the administration of methylphenidate is often the method of choice (e.g., Ritalin, Concerta, Equasym, Medikinet); however, D-amphetamine, as well as non-psychostimulants, such as atomoxetine and guanfacine, are prescribed too [7]. Over the past years, the Multimodal Treatment Study of Children with ADHD and follow-up studies (the so-called MTA studies) have provided ample research regarding stimulant medication, behavioral treatments, their combination, and self-chosen community care. Results demonstrate that both stimulant medication and a combined treatment had a clear clinical benefit in the short term, but on the long-term group differences attenuate, as assessed after 24 months, as well as after 6 and 8 years [8]. These findings, in combination with studies indicating the potential side effects of pharmacotherapy $[9 \cdot, 10]$, partial drug response [7], and the time and cost intensiveness of combining treatments due to the involvement of multiple professionals [6], have resulted in a growing interest into the development of alternative non-pharmacological treatments in ADHD.

For instance, computerized cognitive-based training approaches (e.g., working-memory and attention training) aim to reduce ADHD core symptoms and tackle neuropsychological functioning. Research into this topic is still in the early stages and more controlled studies regarding the effects on ADHD core symptoms are required [11]. Another alternative treatment method for ADHD which is already more extensively studied in the past is neurofeedback. In the following paragraphs, we will (i) introduce neurofeedback, (ii) present standard protocols for ADHD, (iii) review the past and current evidence in the treatment of ADHD, and (iv) depict the current status of institutional and professional regulation of the clinical implementation of neurofeedback.

\section{Definition, History, and Mechanism of Action of} Neurofeedback Despite the recent popularity of neuromodulation techniques, neurofeedback is for the most part still an unknown territory. Neurofeedback is based on a brain-computer interface (BCI) and is implemented by a software system and a processing pipeline, altogether consisting of five elements (Fig. 1) [12•]. Neurofeedback measures the participant's own brain activity, which is pre-processed (steps 1 and 2). Pre-selected brain parameters (a specific frequency band or a brain potential) are calculated online (step 3) and translated to signals that are fed back to the user in real time (step 4). Thus, selected features of brain activity are made perceivable for the participant. Through this feedback, the participant (step 5) can learn to self-regulate his own brain activity to directly alter the underlying neural mechanism of cognition and behavior.

It has been proposed that neurofeedback is based on principles of operant conditioning and procedural skills learning. Due to these learning mechanisms, neuroplasticity is expected to take place during neurofeedback training either via Hebbian plasticity or anti-Hebbian/homeostatic plasticity. Such intrinsic regulatory mechanisms are believed to prevent extreme states of brain activity, such as pathologically high or low synaptic strengths or oscillatory states; for further reading, see [13•].

Nowadays, neurofeedback is used in three ways: (i) as a therapeutic tool to normalize deviating brain activity and treat neurocognitive disorders, (ii) as a so-called peak performance training to enhance cognitive performance in healthy participants, and (iii) as an experimental method to investigate the causal role of neural oscillations in cognition and behavior. More precisely, the neurofeedback research is dominated by two streams: clinical research and neuroscientific inspired research, which is mainly based on recent methodological and technical innovations, as well as on an increasing knowledge about the neural correlates of behavior and cognition. Some examples of recently developed EEG neurofeedback protocols are the upregulation or downregulation of high alpha $[14,15]$, the upregulation of frontal beta [16], and frontal midline theta [17], but also neurofeedback protocols using fMRI neurofeedback [18•].

Historically, neurofeedback dates back to the initial discovery of the human electroencephalogram (EEG) by Hans Berger. Only 6 years after this breakthrough, two French researchers - Gustave Durup and Alfred Fessard-first reported that the EEG alpha rhythm could be subject to classical conditioning [19], which is thought to be one of the basic premises of neurofeedback. This initial observation was followed up by more systematic studies in the early 1940s that further demonstrated all of the Pavlovian types of conditioned responses could be demonstrated on the "EEG alpha blocking response". [20] In a follow-up study, Jasper and Shagass [21] investigated further whether participants could also exert voluntary control over this alpha blocking response. In this study, they had participants press a button, which would switch the lights on and off, and use subvocal verbal commands when pressing the button, (e.g., "Block" when pressing the button and "Stop" when releasing the button). After five sessions, the subject was able to voluntarily suppress alpha activity, while the lights were off (a condition where normally synchronous alpha would be present). Despite these early developments, it was only in the 1970s that these same principles were applied more systematically, and the first clinical implications were described in the literature. These developments were 


\section{Neurofeedback}
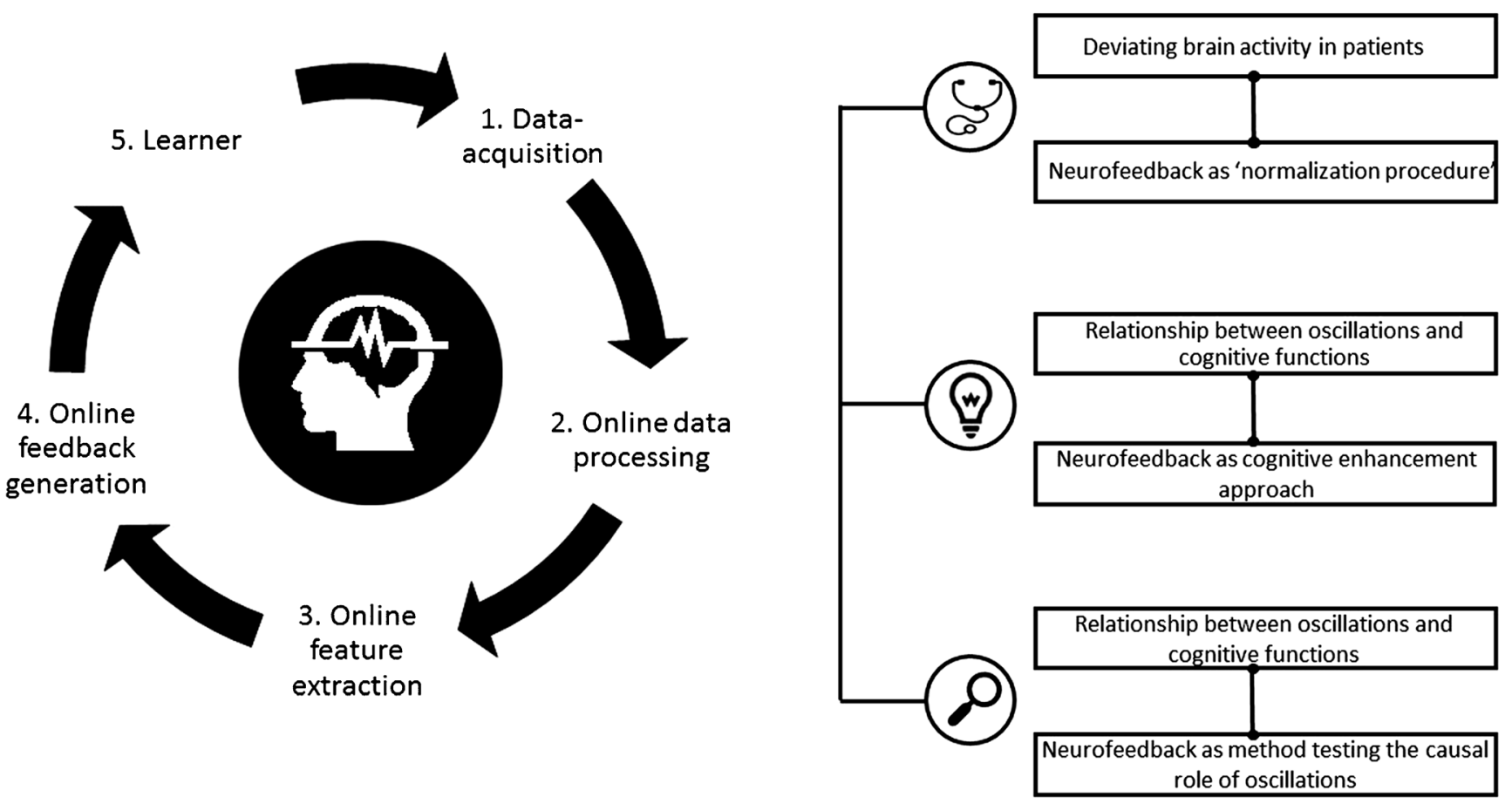

Fig. 1 Overview neurofeedback: neurofeedback pipeline and three areas of neurofeedback application. The pipeline includes the five most important processing steps and parts of a neurofeedback system

motivated by the discovery of the anticonvulsant effects of sensori-motor rhythm (SMR) neurofeedback in cats [22] and subsequently humans [23]. The presumed role of SMR modulation on motor behavior was followed by the first demonstrations of the positive effects of SMR neurofeedback in hyperkinetic disorder [24]. Around the same 1960-1970 period, the first report of voluntary control over a slow brain potential called the contingent negative variation $(\mathrm{CNV})$ or "bereitschaftspotential" (readiness potential, due to the property of this potential to emerge when preparing for action, e.g., when waiting in front of a traffic light) was reported [25], which laid the foundation of another well-known neurofeedback approach, namely of slow cortical potential (SCP) neurofeedback. The first application of SCP neurofeedback in ADHD was reported in 2004 [26]. The initial findings described above as SMR and TBR neurofeedback resulted into what we currently known as "frequency band neurofeedback."

\section{Standard Protocols with ADHD}

Theta/beta $(4-7 \mathrm{~Hz} / 12-21 \mathrm{~Hz})$ ratio (TBR) neurofeedback strives to decrease theta and/or increase beta power in central and frontal locations. This protocol directly targets important electrophysiological characteristics such as high theta/beta ratios, high theta power, and/or low beta power commonly observed in children (for a review, see [27]) and adults with ADHD [28-30]. Recent randomized controlled trials suggest that 30 to 40 sessions of TBR neurofeedback were as effective as methylphenidate in reducing inattentive and hyperactivity symptoms and were even associated with superior posttreatment academic performance [31, 32]. It has been proposed that the effects of TBR neurofeedback on ADHD might be explained by the learned self-regulation of attention [33] as evidenced by enhanced amplitude of endogenous evokedrelated potentials such as the P300 [34]. However, more neuroscientific evidence is needed to determine the specific mechanisms by which TBR neurofeedback might impact cognitive functioning in ADHD.

SMR neurofeedback training over the sensori-motor strip (predominantly in the central right hemispheric region) was first applied to ADHD children by Lubar and colleagues [24, 35], based on the functional association of the sensori-motor rhythm with behavioral inhibition and the promising results in reducing cortical excitability in epileptics obtained by Sterman, MacDonald, and Stone [36]. Lubar's seminal studies revealed that the beneficial hyperactivity-reducing effects of a combined SMR/theta neurofeedback training were maintained after psychostimulants was withdrawn in hyperactive children.

Studies suggest that SMR neurofeedback training reduces inattentive and hyperactive/impulsive symptoms in ADHD children to the same extent as TBR training and comparable 
number of treatment sessions. However, the two protocols might achieve the same results through distinct mechanisms. Arns, Feddema, and Kenemans [37] provided evidence that ADHD patients trained with the SMR protocol showed decreased sleep onset latency (SOL) and improved sleep quality in comparison to those administered with TBR, midway treatment. A mediation analysis revealed that this normalized sleep mid-treatment was responsible for the improved inattention post-treatment. The improvements in ADHD symptoms following SMR training might hence be the result of the vigilance stabilization mediated by the regulation of the locus coeruleus noradrenergic system of which activation has been shown to impact the sleep spindle circuitry [38]. This explanation seems to be in line with previous indications that patients with ADHD present delays in SOL [39] and that SMR training increases sleep spindle density and improves sleep quality in healthy adults [40].

Another standard protocol is the self-regulation of SCP [41, $42 \cdot \bullet$ ] after around 35 sessions. SCP neurofeedback is based on the learned self-regulation of cortical activation and inhibition which are associated with the electrical negativation and positivation of slow cortical electrical deflections respectively. These periodical shifts from electrical positivity to negativity have been described as a phasic tuning mechanism in the regulation of attention [43] as shown by the enhanced reaction time, stimulus detection, and short-term memory during the negative shift phase [44]. Since SCP, of which the CNV is an example, are closely associated with preparatory motor responses with a maximal topographic representation in the motor areas, the vertex is usually the site of choice for training. Differently from TBR and SMR protocols which are typically unidirectional (i.e., instructions either require the participant to increase or decrease the power of the EEG parameter), the self-regulation of SCP usually involves the training in generating both cortical activation and inhibition. In the case of $\mathrm{ADHD}$, the therapeutic focus is on promoting an increase in the firing probabilities of the underlying cortical areas (i.e., negativation). Another difference relative to frequency neurofeedback is that in SCP neurofeedback the learning trials are higher in number and considerably shorter in duration. Interestingly, it has been hypothesized that SCP might also be associated with improvements in sleep. The generation of slow oscillations, in particular negative slow direct current, shifts training during SCP neurofeedback, might exert control over the sleep spindle circuit and therefore facilitate the transition from wakefulness to sleep [45].

\section{Current Status of Efficacy of Standard Protocols for Neurofeedback in ADHD}

As with any emerging new treatments, knowledge of technical aspects of the treatment, proper standards, and education are crucial for appropriately evaluating the merits and pitfalls of neurofeedback. Unfortunately, the unfounded assumption that "neurofeedback = neurofeedback" is often made. Neurofeedback can differentially impact brain functioning depending on the kind protocol and implementation the same way as different pharmacological treatments do (e.g., antidepressants and analgesic drugs). As an illustration, neurofeedback treatments such as the earlier mentioned SMR, TBR, and SCP neurofeedback are well-investigated and effective in the treatment of ADHD while other approaches such as posterior alpha enhancement have been found to be not effective (for a review, see [3].

Especially when restricted to standard protocols such as TBR, SMR, and SCP protocols [3], neurofeedback is a well-investigated treatment for ADHD. This has become evident from several meta-analyses [2, 46••, 47], including a critical meta-analysis from the European ADHD Guidelines Group (EAGG) that also conducted a sensitivity analysis focused on so called "blinded" ratings (i.e., teacher reports only) [4•]. Blinded ratings have usually lower effects sizes than ratings by people most-proximal to the child and therefore least blinded (e.g., parents) and both rating types are only modestly correlated [48]. One explanation for this may be that the rating types focus on different aspects of ADHD symptoms. This is reflected in studies showing different rating-ADHD aspect associations, as for instance parent ratings of hyperactiveimpulsive behaviors were found to be correlated with genetics [49], whereas teacher ratings have been shown to be associated to medication effects [50], most likely due to the fast onset of action of psychostimulants. To come back to the latter meta-analysis [4•], the researchers did not find an effect of neurofeedback in general on teacher-rated ADHD symptoms, but there was an effect when the analysis was restricted to the above mentioned "standard protocols." Finally, a recent meta-analysis that included 10 RCTs and specifically looked at long-term effects of neurofeedback, compared to active treatments (including psychostimulants) and semi-active treatments (e.g., cognitive training), found that after on average 6 months followup, the effects of neurofeedback were superior to semiactive control groups and no different from active treatments including methylphenidate [46••]. Interestingly, this meta-analysis confirmed the trend for medication effects to diminish with time, and the effects of neurofeedbackwithout additional sessions being conducted - to increase with time. These data suggest the promising aspect, namely of long-term efficacy, of neurofeedback. Currently, one of the largest and most comprehensive double-blind multisite RCT is carried out: the International Collaborative ADHD Neurofeedback study (ICAN). This study consists of a cross-site investigation team with different background of ADHD treatment approaches assessing 140 
participants in total (see the study design in [51]), and results are foreseen to be published in 2019 .

\section{Current Status of Institutional and Professional Regulation of Clinical Neurofeedback Implementations}

Although standard protocols turn out to be efficacious and specific, the practical implementation of neurofeedback as a clinical therapy is currently not regulated. This applies to the educational standards, medical security, and the usage of standard protocols indicated for specific disorders such as ADHD. The lack of regulation and agreed upon standards comes with the danger of patients being treated with ineffective neurofeedback protocols applied by unlicensed personal (or even worse by people without any health-related background). For instance, although practitioners should stick to standard protocols with functional specificity of the frequency and topographic locations, clinical practice often deviates from what is recommended by research. The lack of regulation and missing standards have furthermore caused a surge in commercial driven applications and proclaimed "innovations" of neurofeedback protocols and implementations. Several studies have now demonstrated that some of those "innovations" and implementations do not work. One example of such ineffective technique is the SmartBrain neurofeedback approach using the "NASA patented engagement index" with Sony PlayStation feedback [51, 52]. Additionally, there is no evidence in favor of the efficacy of unconventional neurofeedback protocols used in some neurofeedback clinics [53] and frequently advertised applications such as $Z$ score and LORETA neurofeedback [54]. Unfortunately, these proclaimed innovations and commercialdriven applications only add noise to the ongoing debate of neurofeedback efficacy and risk "throwing the baby out with the bathwater." However, above all this demonstrates the need for further research into the effectiveness of already available and newly developed neurofeedback protocols (i.e., the number of sessions, targeted brain area, selected brain parameter, working mechanism) in addition to proper "agreed-upon standards" and training within the field of neurofeedback.

Neurofeedback researchers and practitioners can affiliate to scientific and professional organizations at the international and national level. On an international level, there are mainly two societies. The Society of Applied Neuroscience (SAN) (http://www.applied-neuroscience. org/) is an EU-based nonprofit membership organization for the advancement of neuroscientific knowledge and development of innovative applications for optimizing brain functioning (such as neurofeedback with EEG, fMRI, NIRS). The International Society for Neurofeedback \& Research (ISNR (https://www.isnr.org) is a membership organization aimed at supporting scientific research in applied neurosciences, promoting education in the field of neurofeedback, albeit not always clearly separating commercial and objective interests. Other neurofeedback societies or organizations are often connected to certain neurofeedback equipment manufacturers and have (seemingly) conflicting interests. Furthermore, the Biofeedback Certification International Alliance (BCIA) is a broader international licensure also including biofeedback (www.BCIA.org).

\section{Conclusions}

Recent years witness a renewed interest in neurofeedback in response to the lack of long-term effects for both medication and behavioral therapy and the side effects of medication. Herein, we provide evidence for the efficacy and specificity of standard neurofeedback protocols, namely theta/beta, sensori-motor rhythm, and slow cortical potential. In line with the guidelines for rating evidence developed by the APA, "standard" neurofeedback protocols have been considered to be "Efficacious and Specific, Level V" in the treatment of ADHD (AAPB Guidelines: [57]).

However, currently there are no uniform standards regarding training courses for neurofeedback that are accepted by expert associations, neither national-wide, nor in the EU or USA. While performing neurofeedback in a therapeutic context, a thorough basic training, a distinct technical understanding of the medical devices, the software, and the EEG caps, as well as continuing education, are imperative. Regarding the medical security performing neurofeedback in a clinical context, neurofeedback devices (hardware: amplifier and EEG caps, neurofeedback software) are neither regulated in a strict way. However, it is essential that besides the absolute minimum technical requirements after the Medical Device Regulation (MDR) EU 2017/745), neurofeedback devices should be regulated by both the $\mathrm{CE}$ (that confirms a medical device meets the essential MDR requirements) and a European equivalent of the Food and Drug Administration (FDA). The FDA enforces laws to protect the consumer's health, safety, and pocketbook. Such potential regulating mechanisms could be implemented by the European medicine regulatory network. In short, tasks ahead concern regulating neurofeedback as therapy, developing internationally accepted binding standards for education and NF implementation and the qualification of neurofeedback trainers.

Last but not least, Brian — now 4 years later - discontinued his medication successfully under medical supervision. Due to neurofeedback, his impulsivity symptoms strongly reduced and he gained control over his concentration, doing well in high school performance. 


\section{Compliance with Ethical Standards}

Conflict of Interest Stefanie Enriquez-Geppert and Diede Smit each declare that they have no conflict of interest. Miguel G Pimenta declares that he is a lecturer in neurofeedback for the neuroCare Group (Munich, Germany). Martijn Arns (MAr) reports research grants and options from Brain Resource (Sydney, Australia); owns stock in and serves as Chief Scientific Adviser of the neuroCare Group (Munich, Germany) and Director and Researcher of Research Institute Brainclinics (Nijmegen, Netherlands); is a consultant on a National Institute of Mental Health, US-funded iCAN study (CNG 2013); and is a co-inventor on four patent applications (A61B5/0402; US2007/0299323, A1; WO2010/139361 A1; one pending) related to EEG, neuromodulation, and psychophysiology (not related to neurofeedback). MAr declares no ownership or financial gains for these patents - just authorship.

Human and Animal Rights and Informed Consent This article does not contain any studies with human or animal subjects performed by any of the authors.

Open Access This article is distributed under the terms of the Creative Commons Attribution 4.0 International License (http:// creativecommons.org/licenses/by/4.0/), which permits unrestricted use, distribution, and reproduction in any medium, provided you give appropriate credit to the original author(s) and the source, provide a link to the Creative Commons license, and indicate if changes were made.

\section{References}

Papers of particular interest, published recently, have been highlighted as:

- Of importance

•. Of major importance

1. Hammond DC, Kirk L. Negative effects and the need for standards of practice in neurofeedback. Biofeedback. 1982;35(4):139-45.

2. Arns M, de Ridder S, Strehl U, Breteler M, Coenen A. Efficacy of neurofeedback treatment in ADHD: the effects on inattention, impulsivity and hyperactivity: a meta-analysis. Clin EEG Neurosci. 2009;40(3):180-9.

3. Arns M, Heinrich H, Strehl U. Evaluation of neurofeedback in ADHD: the long and winding road. Biol Psychol. 2014;95:108-15.

4. Cortese S, Ferrin M, Brandeis D, Holtmann M, Aggensteiner P, Daley D, et al. Neurofeedback for attention-deficit/hyperactivity disorder: meta-Analysis of clinical and neuropsychological outcomes from randomized controlled trials. J Am Acad Child Adolesc Psychiatry. 2016;55(6):444-55. This meta-analysis shows that there is only an effect on blinded teacher ratings for ADHD symptoms for standard protocols, but not when assessing all available neurofeedback protocols.

5. Taylor E, Dopfner M, Sergeant J. European clinical guidelines for hyperkinetic disorder - first upgrade. Eur Child Adolesc Psychiatry. 2004;13(Suppl 1):i7-i30.

6. Daly BP, Creed T, Xanthopoulos M, Brown RT. Psychosocial treatments for children with attention deficit/hyperactivity disorder. Neuropsychol Rev. 2007;17(1):73-89.

7. Faraone SV, Buitelaar J. Comparing the efficacy of stimulants for ADHD in children and adolescents using meta-analysis. Eur Child Adolesc Psychiatry. 2010;19(4):353-64.

8. Molina BSG, Hinshaw SP, Swanson JM, Arnold LE, Vitiello B, Jensen PS, et al. The MTA at 8 years: prospective follow-up of children treated for combined-type ADHD in a multisite study. J Am Acad Child Adolesc Psychiatry. 2009;48(5):484-500.

9. Storebø OJ, Ramstad E, Krogh HB, Nilausen TD, Skoog M, Holmskov M, et al. Methylphenidate for children and adolescents with attention deficit hyperactivity disorder (ADHD). Cochrane Database Syst Rev. 2015;11:CD009885. In this article, the side effects of methylphenidate are systematically presented.

10. Charach A, Figueroa M, Chen S, Ickowicz A, Schachar R. Stimulant treatment over 5 years: effects on growth. J Am Acad Child Adolesc Psychiatry. 2006;45(4):415-21.

11. Sonuga-Barke EJ, Brandeis D, Holtmann M, Cortese S. Computerbased cognitive training for attention-deficit/hyperactivity disorder: a review of current evidence. Child Adolesc Psychiatr Clin N Am. 2014;23(4):807-24.

12. Enriquez-Geppert S, Huster RJ, Herrmann CS. EEGneurofeedback as a tool to modulate cognition and behavior: a review tutorial. Front Hum Neurosci. 2017;11:51. This review gives an overview of the key aspects relevant to the development of neurofeedback protocols and discusses the relevant aspects for the practical application of neurofeedback.

13. Ros T, Baars BJ, Lanius RA, Vuilleumier P. Tuning pathological brain oscillations with neurofeedback: a systems neuroscience framework. Front Hum Neurosci. 2014;8:1008. This article includes a section about the currently known mechanisms of actions of neurofeedback.

14. Escolano C, Aquilar M, Minguey J. EEG-based upper alpha neurofeedback training improves working memory performance. Conf Proc IEEE Eng Med Biol Soc. 2011:2327-30.

15. Ros T, Munneke M, Ruge D, Gruzelier J, Rothwell J. Endogenous control of waking brain rhythms induces neuroplasticity in humans. Eur J Neurosci. 2010;31(4):770-8.

16. Engelbregt HJ, Keeser D, Van Eijk L, Suiker EM, Eichhorn D, Karch S, et al. Short and long-term effects of sham-controlled prefrontal EEG-neurofeedback training in healthy subjects. Clin Neurophysiol. 2016;127(4):1931-7.

17. Enriquez-Geppert S, Huster RJ, Figge C, Herrmann CS. Selfregulation of frontal-midline theta facilitates memory updating and mental set shifting. Front Behav Neurosci. 2014;8:420.

18. Alegria AA, Wulff M, Brinson H, Barker GJ, Norman LJ, Brandeis $\mathrm{D}$, et al. Real-time fMRI neurofeedback in adolescents with attention deficit hyperactivity disorder. Hum Brain Mapp. 2017;38(6): 3190-209. This article presents the effects of fMRI neurofeedback on dysregulated brain regions in ADHD.

19. Durup G, Fessard A. I. L'électrencéphalogramme de l'homme. Observations psycho-physiologiques relatives à l'action des stimuli visuels et auditifs. Ann Psychol. 1935;36(1):1-32.

20. Jasper H, Shagass C. Conditioning the occipital alpha rhythm in man. J Exp Psychol. 1941;28(5):373-88.

21. Jasper H, Shagass C. Conscious time judgments related to conditioned time intervals and voluntary control of the alpha rhythm. J Exp Psychol. 1941;28(6):503-8.

22. Sterman B, LoPresti RW, Fairchild MD. Electroencephalographic and behavioral studies of monomethylhydrazine toxicity in the cat. J Neurother. 2010;14(4):293-300.

23. Sterman MB, Friar L. Suppression of seizures in an epileptic following sensorimotor EEG feedback training. Electroencephalogr Clin Neurophysiol. 1972;33(1):89-95.

24. Lubar JF, Shouse MN. EEG and behavioral changes in a hyperkinetic child concurrent with training of the sensorimotor rhythm (SMR). Biofeedback Self Regul. 1976;1(3):293-306.

25. McAdam DW, Irwin DA, Rebert CS, Knott JR. Conative control of the contingent negative variation. Electroencephalogr Clin Neurophysiol. 1966;21(2):194-5.

26. Heinrich H, Gevensleben H, Freisleder FJ, Moll GH, Rothenberger A. Training of slow cortical potentials in attention-deficit/ 
hyperactivity disorder: evidence for positive behavioral and neurophysiological effects. Biol Psychiatry. 2004;55(7):772-5.

27. Barry RJ, Clarke AR, Johnstone SJ. A review of electrophysiology in attention-deficit/hyperactivity disorder: I. Qualitative and quantitative electroencephalography. Clin Neurophysiol. 2003;114(2):171-83.

28. Bresnahan SM, Barry RJ. Specificity of quantitative EEG analysis in adults with attention deficit hyperactivity disorder. Psychiatry Res. 2002;112(2):133-44.

29. Bresnahan SM, Anderson JW, Barry RJ. Age-related changes in quantitative EEG in attention-deficit/hyperactivity disorder. Biol Psychiatry. 1999;46(12):1690-7.

30. Clarke AR, Barry RJ, Heaven PCL, McCarthy R, Selikowitz M, Byrne MK. EEG in adults with attention-deficit/hyperactivity disorder. Int J Psychophysiol. 2008;70(3):176-83.

31. Duric NS, Assmus J, Gundersen D, Elgen IB. Neurofeedback for the treatment of children and adolescents with ADHD: a randomized and controlled clinical trial using parental reports. BMC Psychiatry. 2012;12:107.

32. Meisel V, Servera M, Garcia-Banda G, Cardo E, Moreno I. Neurofeedback and standard pharmacological intervention in ADHD: a randomized controlled trial with six-month follow-up. Biol Psychol. 2013;94(1):12-21.

33. Gevensleben H, Rothenberger A, Moll GH, Heinrich H. Neurofeedback in children with ADHD: validation and challenges. Expert Rev Neurother. 2012;12(4):447-60.

34. Egner T, Gruzelier JH. EEG biofeedback of low beta band components: frequency-specific effects on variables of attention and eventrelated brain potentials. Clin Neurophysiol. 2004;115(1):131-9.

35. Shouse MN, Lubar JF. Operant conditioning of EEG rhythms and Ritalin in the treatment of hyperkinesis. Biofeedback Self Regul. 1979;4(4):299-312.

36. Sterman MB, Macdonald LR, Stone RK. Biofeedback training of the sensorimotor electroencephalogram rhythm in man: effects on epilepsy. Epilepsia. 1974;15(3):395-416.

37. Arns M, Feddema I, Kenemans JL. Differential effects of theta/beta and SMR neurofeedback in ADHD on sleep onset latency. Front Hum Neurosci. 2014a;8:1019.

38. Sinha SR. Basic mechanisms of sleep and epilepsy. J Clin Neurophysiol. 2011;28(2):103-10.

39. Van Veen MM, Kooij JJS, Boonstra AM, Gordijn MCM, Van Someren EJW. Delayed circadian rhythm in adults with attentiondeficit/hyperactivity disorder and chronic sleep-onset insomnia. Biol Psychiatry. 2010;67(11):1091-6.

40. Schabus M, Heib DPJ, Lechinger J, Griessenberger H, Klimesch W, Pawlizki A, et al. Enhancing sleep quality and memory in insomnia using instrumental sensorimotor rhythm conditioning. Biol Psychol. 2014;95:126-34.

41. Gevensleben H, Holl B, Albrecht B, Schlamp D, Kratz O, Studer P, et al. Distinct EEG effects related to neurofeedback training in children with ADHD: a randomized controlled trial. Int J Psychophysiol. 2009;74(2):149-57.

42.• Strehl U, Aggensteiner P, Wachtlin D, Brandeis D, Albrecht B, Arana M, et al. Neurofeedback of slow cortical potentials in children with attention-deficit/hyperactivity disorder: a multicenter randomized trial controlling for unspecific effects. Front Hum
Neurosci. 2017;11:135. This multi-center RCT shows clinical efficacy of standard neurofeedback protocols for ADHD.

43. Rockstroh B, Elbert T, Birbaumer N, Lutzenberger W. Biofeedbackproduced hemispheric asymmetry of slow cortical potentials and its behavioural effects. Int J Psychophysiol. 1990;9(2):151-65.

44. Birbaumer N, Elbert T, Canavan AG, Rockstroh B. Slow potentials of the cerebral cortex and behavior. Physiol Rev. 1990;70(1):1-41.

45. Marshall L, Mölle M, Born J. Spindle and slow wave rhythms at slow wave sleep transitions are linked to strong shifts in the cortical direct current potential. Neuroscience. 2003;121(4):1047-53.

46.• Van Doren J, Arns M, Heinrich H, Vollebregt MA, Strehl U, Loo SK. Sustained effects of neurofeedback in ADHD: A systematic review and meta-analysis. Eur Child Adolesc Psychiatry. 2018:113. This recent meta-analysis with 10 RCTs shows that the effects of neurofeedback increase in time, other than medication effects that diminish with time, and suggest of long-term efficacy of neurofeedback.

47. Micoulaud-Franchi JA, Geoffroy PA, Fond G, Lopez R, Bioulac S, Philip P. EEG neurofeedback treatments in children with ADHD: an updated meta-analysis of randomized controlled trials. Front Hum Neurosci. 2014;8:906.

48. Sollie H, Larsson B, Morch WT. Comparison of mother, father, and teacher reports of ADHD core symptoms in a sample of child psychiatric outpatients. J Atten Disord. 2013;17(8):699-710.

49. Bralten J, Franke B, Waldman I, Rommelse N, Hartman C, Asherson $\mathrm{P}$, et al. Candidate genetic pathways for attention deficit/hyperactivity disorder (ADHD) show association to hyperactive/impulsive symptoms in children with ADHD. J Am Acad Child Adolesc Psychiatry. 2013;52(11):1204-12.

50. Wang LJ, Chen C-K, Huang YS. Changes in behaviour symptoms of patients with attention deficit/hyperactivity disorder during treatment: observation from different informants. Psychiatry Investig. 2013;10(1):1-7.

51. Arnold LE, Lofthouse N, Hersch S, Pan X, Hurt E, Bates B, et al. EEG neurofeedback for ADHD: double-blind sham-controlled randomized pilot feasibility trial. J Atten Disord. 2013;17(5):410-9.

52. DeBeus R, Kaiser D. Neurofeedback with children with attention deficit hyperactivity disorder: a randomized double-blind placebocontrolled study. In: Coben R, Evans J, editors. Neurofeedback and neuromodulation: techniques and applications, vol. 2011. San Diego: Elsevier; 2011. p. 127-52.

53. Van Dongen-Boomsma M, Vollebregt MA, Slaats-Willemse D, Buitelaar J. A randomized placebo-controlled trial of electroencephalographic (EEG) neurofeedback in children with attentiondeficit/hyperactivity disorder. J Clin Psychiatry. 2013;74(8):821-7.

54. Coben R, Hammond DC, Arns M. 19 channel z-score and LORETA neurofeedback: does the evidence support the hype? Appl Psychophysiol Biofeedback. 2018:1-8.

Publisher's Note Springer Nature remains neutral with regard to jurisdictional claims in published maps and institutional affiliations. 